06

\title{
Генерация второй гармоники от тонкого цилиндрического слоя. II. Анализ решения
}

\author{
(c) В.Н. Капшай, А.А. Шамына
}

Гомельский государственный университет им. Ф. Скорины, 246019 Гомель, Беларусь

e-mail: anton.shamyna@gmail.com; kapshai@rambler.ru

Поступила в редакцию 24.12.2018 г.

В окончательной редакции 16.01.2019 г.

Принята к публикации 23.01.2019 г.

\begin{abstract}
Проанализировано решение задачи о генерации второй гармоники плоской электромагнитной волной с эллиптической поляризацией от тонкого оптически нелинейного слоя на поверхности цилиндрической частицы конечных размеров. Проведен графический анализ решения путем построения трехмерных диаграмм направленности, характеризующих пространственное распределение мощности излучения второй гармоники и его поляризацию. Обнаружено, что при малых радиусах основания цилиндрической частицы каждому коэффициенту анизотропии соответствует своя индивидуальная форма диаграммы направленности. Увеличение высоты цилиндрической частицы ведет к сплющиванию диаграммы направленности. Найдено более 20 математических свойств решения, характеризующих симметрию распределения поля второй гармоники. Отдельные свойства проиллюстрированы на диаграммах направленности.
\end{abstract}

DOI: $10.21883 /$ OS.2019.06.47766.375-18

\section{Анализ решения}

\section{Явный вид векторов в задаче}

Настоящая работа является второй частью статьи о генерации второй гармоники от поверхности диэлектрических частиц цилиндрической формы.

Анализ решения задачи о генерации второй гармоники от поверхности цилиндрической частицы позволяет выделить характерные для отдельных комбинаций параметров задачи симметрии и свойства диаграмм направленностей генерируемого излучения. Это дает возможность по форме диаграммы направленности оценить доминирующий тип анизотропии и размеры частиц.

Не нарушая общности, будем считать, что волновой вектор падающей волны $\mathbf{k}^{(\omega)}$ направлен так, что его составляющая (если она есть) в плоскости $O x y$ направлена вдоль оси $O x$. Тогда векторы в задаче выражаются через базисные векторы в следующем виде:

$$
\begin{aligned}
\mathbf{k}^{(\omega)} & =k_{\omega} \sin \theta_{i n} \mathbf{e}_{x}+k_{\omega} \cos \theta_{i n} \mathbf{e}_{z}, \\
\mathbf{e}^{(\omega)}= & \frac{\cos \theta_{i n}\left(-\cos \varphi_{i n}+i \sigma \sin \varphi_{i n}\right)}{\sqrt{1+\sigma^{2}}} \mathbf{e}_{x} \\
& +\frac{\left(i \sigma \cos \varphi_{i n}-\sin \varphi_{i n}\right)}{\sqrt{1+\sigma^{2}}} \mathbf{e}_{y} \\
& +\sin \theta_{i n} \frac{\cos \varphi_{i n}-i \sigma \sin \varphi_{i n}}{\sqrt{1+\sigma^{2}}} \mathbf{e}_{z} .
\end{aligned}
$$

Выражение для составляющей вектора рассеяния $\mathbf{q}_{\|}$:

$$
\begin{aligned}
\mathbf{q}_{\|} & =\mathbf{e}_{z}\left[\mathbf{e}_{z}\left(2 \mathbf{k}^{(\omega)}-\mathbf{k}^{(2 \omega)}\right)\right] \\
& =\mathbf{e}_{z} 2 k_{\omega}\left(\cos \theta_{i n}-\xi \cos \theta\right)=\mathbf{e}_{z} q_{\|}, \quad \xi=k_{2 \omega} /\left(2 k_{\omega}\right),
\end{aligned}
$$

Перпендикулярная составляющая вектора рассеяния с учетом (1) запишется в виде

$$
\begin{aligned}
\mathbf{q}_{\perp}= & \left(1-\mathbf{e}_{z} \otimes \mathbf{e}_{z}\right)\left(2 \mathbf{k}^{(\omega)}-\mathbf{k}^{(2 \omega)}\right) \\
= & -2 k_{\omega}\left[\left(\xi \sin \theta-\sin \theta_{i n} \cos \varphi\right) \mathbf{e}_{\rho}+\sin \theta_{i n} \sin \varphi \mathbf{e}_{\varphi}\right], \\
q_{\perp}= & \left|\mathbf{q}_{\perp}\right|=2 k_{\omega} \\
& \times \sqrt{\sin ^{2} \theta_{i n}-2 \xi \sin \theta_{i n} \sin \theta \cos \varphi+\xi^{2} \sin ^{2} \theta} .
\end{aligned}
$$

Отметим, что $q_{\perp}=0$, если $\sin ^{2} \theta_{i n}-2 \xi \sin \theta_{i n} \sin \theta \cos \varphi$ $+\xi^{2} \sin ^{2} \theta=0$. Если $q_{\perp} \neq 0$, то можно ввести единичный вектор вдоль $\mathbf{q}_{\perp}$ :

$$
\boldsymbol{v}=\frac{\mathbf{q}_{\perp}}{\left|\mathbf{q}_{\perp}\right|}=\frac{\left(-\xi \sin \theta+\sin \theta_{i n} \cos \varphi\right) \mathbf{e}_{\rho}-\sin \theta_{i n} \sin \varphi \mathbf{e}_{\varphi}}{\sqrt{\sin ^{2} \theta_{i n}-2 \xi \sin \theta_{i n} \sin \theta \cos \varphi+\xi^{2} \sin ^{2} \theta}}
$$

В случае, когда $q_{\perp}=0$, будем считать, что $v=0$. Все векторы в задаче изображены схематично на рис. $1, a, b$.

\section{Свойства функций, характеризующих пространственное распределение поля второй гармоники}

Используя формулы (1), (2) и формулы (15), (28), (29) из части $\mathrm{I}$, можно получить угловые зависимости $\mathbf{f}^{(2 \omega)}(\theta, \varphi)$ и $S_{r}^{(2 \omega)}(\theta, \varphi)$. Анализ математических свойств этих функций позволяет выявить возникающую симметрию в диаграммах направленности при графическом анализе. Проявление подобных свойств на эксперименте можно наблюдать при генерации второй гармоники от поверхности сферических частиц [1-4] (плоскость 
$a$

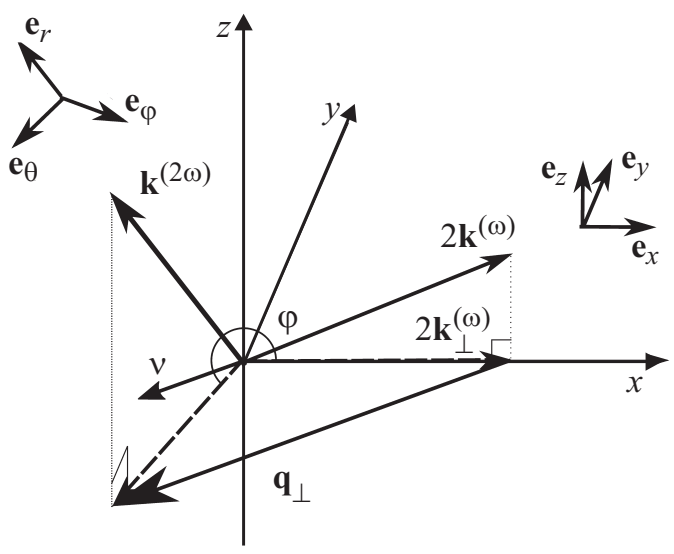

$b$

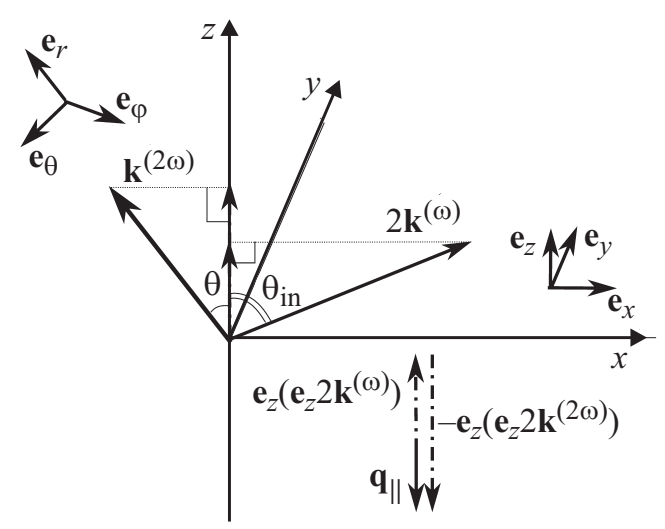

Рис. 1. Схема задачи о генерации второй гармоники от поверхности цилиндрической частицы: $a-$ схема векторов, используемых для получения $\mathbf{q}_{\perp} ; b-$ схема векторов, используемых для получения $\mathbf{q}_{\|}$. Штриховые стрелки обозначают векторы в плоскости $O x y$. Штрихпунктирные стрелки обозначают векторы вдоль оси $O z$.

симметрии между главными лепестками диаграммы направленности). Далее приведем обнаруженные математические свойства функций $\mathbf{f}^{(2 \omega)}(\theta, \varphi)$ и $S_{r}^{(2 \omega)}(\theta, \varphi)$.

При любых целых $m_{1}, m_{2}$ выполняются равенства

$$
\begin{gathered}
\mathbf{f}^{(2 \omega)}\left(\theta+2 \pi m_{1}, \varphi+2 \pi m_{2}\right)=\mathbf{f}^{(2 \omega)}(\theta, \varphi), \\
\mathbf{f}^{(2 \omega)}(-\theta, \varphi)=-\left(1-2 \mathbf{e}_{r} \otimes \mathbf{e}_{r}\right) \mathbf{f}^{(2 \omega)}(\theta, \varphi+\pi), \\
S_{r}^{(2 \omega)}\left(\theta+2 \pi m_{1}, \varphi+2 \pi m_{2}\right)=S_{r}^{(2 \omega)}(\theta, \varphi), \\
S_{r}^{(2 \omega)}(-\theta, \varphi)=S_{r}^{(2 \omega)}(\theta, \varphi+\pi) .
\end{gathered}
$$

Эти общие свойства работают и для генерации второй гармоники от тонкого слоя на поверхности сферической частицы [5], а также рассмотрении генерации суммарной частоты от тонкого слоя на поверхности цилиндрической [6] или сферической частицы $[7,8]$.

Для типа анизотропии $\chi_{2}^{(2)} \neq 0, \chi_{1,3,4}^{(2)}=0$ выполняются равенства

$$
\begin{gathered}
\mathbf{f}^{(2 \omega)}(\theta,-\varphi)=\left(1-2 \mathbf{e}_{\varphi} \otimes \mathbf{e}_{\varphi}\right) \mathbf{f}^{(2 \omega)}(\theta, \varphi), \\
\operatorname{Re}\left[\mathbf{f}^{(2 \omega)}(\theta, \varphi)\right]=0, \\
S_{r}^{(2 \omega)}(\theta,-\varphi)=S_{r}^{(2 \omega)}(\theta, \varphi) .
\end{gathered}
$$

Для нормального падения на цилиндрическую частицу $\left(\theta_{i n}=\pi / 2\right)$ проявляются также свойства

$$
\begin{gathered}
\mathbf{f}^{(2 \omega)}(\theta,-\varphi)=-\left(1-2 \mathbf{e}_{r} \otimes \mathbf{e}_{r}\right) \mathbf{f}^{(2 \omega)}(\pi-\theta, \varphi), \\
\mathbf{e}_{\varphi} \mathbf{f}_{l a t}^{(2 \omega)}(\theta,-\varphi)=-\mathbf{e}_{\varphi} \mathbf{f}_{l a t}^{(2 \omega)}(\theta, \varphi), \\
S_{r}^{(2 \omega)}(\theta,-\varphi)=S_{r}^{(2 \omega)}(\pi-\theta, \varphi) .
\end{gathered}
$$

Если большая ось эллипса поляризации перпендикулярна или параллельна плоскости $O x z\left(\varphi_{i n}=\pi m / 2\right.$, где $m$ - целое), то

$$
i \mathbf{f}^{(2 \omega)}(\theta,-\varphi)=\left[i\left(1-2 \mathbf{e}_{\varphi} \otimes \mathbf{e}_{\varphi}\right) \mathbf{f}^{(2 \omega)}(\theta, \varphi)\right]^{*},
$$

$$
S_{r}^{(2 \omega)}(\theta,-\varphi)=S_{r}^{(2 \omega)}(\theta, \varphi) .
$$

Для линейной поляризации падающей волны $(\sigma=0)$, если вектор напряженности электрического поля падающего излучения перпендикулярен плоскости $O x z$ $\left(\varphi_{i n}=m \pi+\pi / 2\right.$, где $m$ - целое $)$, верно следующее

$$
\operatorname{Re}\left[\mathbf{e}_{\varphi} \mathbf{f}^{(2 \omega)}(\theta, \varphi)\right]=0 .
$$

В некиральном случае $\left(\chi_{1-3}^{(2)} \neq 0, \chi_{4}^{(2)}=0\right)$, если $\varphi_{\text {in }}=\pi m / 4$, где $m$ - целое,

$$
\begin{aligned}
& \operatorname{Re}\left[\mathbf{f}^{(2 \omega)}(\theta,-\varphi) \exp (i(m+1) \pi / 2)\right] \\
& =\operatorname{Re}\left[\left(1-2 \mathbf{e}_{\varphi} \otimes \mathbf{e}_{\varphi}\right) \mathbf{f}^{(2 \omega)}(\theta, \varphi) \exp (i(m+1) \pi / 2)\right]
\end{aligned}
$$

При таком же типе анизотропии $\left(\chi_{1-3}^{(2)} \neq 0, \chi_{4}^{(2)}=0\right)$ и линейной поляризации $(\sigma=0)$ выполняется свойство

$$
\operatorname{Re}\left[\mathbf{f}^{(2 \omega)}(\theta, \varphi)\right]=0
$$

Если падающая волна направлена вдоль оси цилиндра $\left(\theta_{\text {in }}=0, \pi\right)$, то

$$
\begin{gathered}
\mathbf{f}^{(2 \omega)}(-\theta, \varphi)=-\left(1-2 \mathbf{e}_{r} \otimes \mathbf{e}_{r}\right) \mathbf{f}^{(2 \omega)}(\theta, \varphi+\pi m), \\
\operatorname{Im}\left[\left(1-(1-i) \mathbf{e}_{\varphi} \otimes \mathbf{e}_{\varphi}\right) \mathbf{f}^{(2 \omega)}\left(\theta, \frac{\pi m}{2}+(-1)^{\frac{\theta_{i n}}{\pi}} \varphi_{i n}-\varphi\right)\right. \\
\quad \times \exp (i m \pi / 2)]=\operatorname{Im}\left[\left(1-(1-i) \mathbf{e}_{\varphi} \otimes \mathbf{e}_{\varphi}\right)\right. \\
\left.\quad \times \mathbf{f}^{(2 \omega)}\left(\theta,(-1)^{\frac{\theta_{i n}}{\pi}} \varphi_{i n}+\varphi\right) \exp (i m \pi / 2)\right], \\
i\left(1-(1-i) \mathbf{e}_{\varphi} \otimes \mathbf{e}_{\varphi}\right) \mathbf{f}^{(2 \omega)}\left(\theta, \pi m-\varphi+(-1)^{\theta_{i n} / \pi} \varphi_{i n}\right) \\
=\left[i\left(1-(1-i) \mathbf{e}_{\varphi} \otimes \mathbf{e}_{\varphi}\right) \mathbf{f}^{(2 \omega)}\left(\theta, \varphi+(-1)^{\theta_{i n} / \pi} \varphi_{i n}\right)\right]^{*}, \\
\quad S_{r}^{(2 \omega)}(-\theta, \varphi)=S_{r}^{(2 \omega)}(\theta, \varphi+\pi m),
\end{gathered}
$$




$$
\begin{aligned}
S_{r}^{(2 \omega)} & \left(\theta, \pi m-\varphi+(-1)^{\theta_{i n} / \pi} \varphi_{i n}\right) \\
& =S_{r}^{(2 \omega)}\left(\theta, \varphi+(-1)^{\theta_{i n} / \pi} \varphi_{i n}\right), m \text { - целое. }
\end{aligned}
$$

При аксиальной симметрии задачи $\left(\theta_{i n}=0, \pi\right.$, $|\sigma|=1)$ пространственное распределение мощности генерируемых волн не зависит от азимутального угла:

$$
\begin{gathered}
\mathbf{f}^{(2 \omega)}(\theta, \pi+\Delta \varphi)=\mathbf{f}^{(2 \omega)}(\theta, \pi m+\varphi) \exp \left(i 2 \sigma(-1)^{\theta_{i n} / \pi} \Delta \varphi\right) \\
i\left(1-(1-i) \mathbf{e}_{\theta} \otimes \mathbf{e}_{\theta}\right) \mathbf{f}^{(2 \omega)}(-\theta, \varphi)= \\
=\left[i\left(1-(1-i) \mathbf{e}_{\theta} \otimes \mathbf{e}_{\theta}\right) \mathbf{f}^{(2 \omega)}(\theta, \pi m-\varphi) \exp \left(4 i \sigma \varphi_{i n}\right)\right]^{*} \\
S_{r}^{(2 \omega)}(\theta, \varphi+\Delta \varphi)=S_{r}^{(2 \omega)}(\theta, \varphi), \\
S_{r}^{(2 \omega)}(-\theta, \varphi)=S_{r}^{(2 \omega)}(\theta, \pi m-\varphi),
\end{gathered}
$$

$\Delta \varphi$ - действительное, $m$ - целое.

При типе анизотропии $\chi_{2}^{(2)} \neq 0, \chi_{1,3,4}^{(2)}=0$ и падении волны вдоль оси цилиндра $\left(\theta_{i n}=0, \pi\right)$ осевая симметрия наблюдается при любой поляризации падающего излучения:

$$
\begin{gathered}
\mathbf{e}_{\varphi} \mathbf{f}^{(2 \omega)}(\theta, \varphi)=0, \quad \mathbf{f}^{(2 \omega)}(\theta, \varphi+\Delta \varphi)=\mathbf{f}^{(2 \omega)}(\theta, \varphi), \\
S_{r}^{(2 \omega)}(\theta, \varphi+\Delta \varphi)=S_{r}^{(2 \omega)}(\theta, \varphi),
\end{gathered}
$$

$\Delta \varphi$ - действительное.

При некиральном типе анизотропии $\left(\chi_{1-3}^{(2)} \neq 0\right.$, $\left.\chi_{4}^{(2)}=0\right)$ и падении вдоль оси цилиндра $\left(\theta_{i n}=0, \pi\right)$ выполняется также свойство

$$
\begin{aligned}
& \operatorname{Re}\left[\mathbf{f}^{(2 \omega)}(\theta, \varphi+\pi m / 2+\pi / 2)\right] \\
& \quad=-(-1)^{m} \operatorname{Re}\left[\mathbf{f}^{(2 \omega)}(\theta, \varphi)\right], m-\text { целое. }
\end{aligned}
$$

А при киральном типе анизотропии $\chi_{1-3}^{(2)}=0, \chi_{4}^{(2)} \neq 0$ и падении вдоль оси цилиндра $\left(\theta_{i n}=0, \pi\right)$ получаем аналогичное свойство:

$$
\begin{aligned}
& \operatorname{Im}\left[\mathbf{f}^{(2 \omega)}(\theta, \varphi+\pi m+\pi / 2)\right] \\
& \quad=-\operatorname{Im}\left[\mathbf{f}^{(2 \omega)}(\theta, \varphi)\right], m-\text { целое. }
\end{aligned}
$$

\section{Графический анализ}

Анализ источников электромагнитных волн с помощью построения их диаграмм направленности неоднократно доказал свою эффективность ввиду своей наглядности. Поэтому применим этот подход для нашей задачи. В диаграммах направленности подразумевается, что в начале координат находится излучающая частица, а лепестки указывают направления, в которых происходит излучение большей части мощности. Этот инструмент также удобен для описания поляризации генерируемых волн. Значение коэффициента $\xi$, характеризующего дисперсию, примем равным единице при построении всех диаграмм направленности.

Наибольшее влияние на форму диаграммы направленности при малых размерах частицы $\left(k_{\omega} a=0.1\right.$, $\left.k_{\omega} h=0.1\right)$ оказывают значения коэффициентов анизотропии $\chi_{1-4}^{(2)}$. На рис. $2, a-d$ изображены трехмерные нормированные диаграммы направленности при генерации от боковой поверхности для случаев, когда все коэффициенты анизотропии, кроме одного, равны нулю. Каждому коэффициенту соответствует своя форма диаграммы направленности. Зная характерные формы, можно определить преобладающее значение коэффициента анизотропии для конкретного исследуемого образца.

На рис. 2,e изображена диаграмма направленности для генерации от торцевых поверхностей. При этом она практически одинакова для случаев, когда $\chi_{1}^{(2)} \neq 0$ и $\chi_{2}^{(2)} \neq 0$. Разнообразие форм диаграмм направленности больше при генерации от боковой поверхности частицы из-за более сложной формы поверхности.

На рис. 3 проанализировано влияние размера цилиндрической частицы на форму диаграммы направленности. Для удобства сравнения выбран случай $\sigma=0.5$, $\theta_{i n}=\pi / 2, \varphi_{\text {in }}=0, \chi_{1}^{(2)} \neq 0, \chi_{2-4}^{(2)}=0$, как на рис. 2,a. На рис. 3, $a$ построена диаграмма для генерации от боковой поверхности цилиндра с большим радиусом основания $\left(k_{\omega} a=2, k_{\omega} h=0.1\right)$. При этом на диаграмме выделяются два главных лепестка и два побочных. При дальнейшем увеличении радиуса основания длина больших лепестков растет, а их ширина уменьшается. Количество побочных лепестков при этом увеличивается. Аналогичные закономерности характерны и для других значений коэффициентов анизотропии.

На рис. 3, $b$ изображена диаграмма для генерации от боковой поверхности цилиндра с большой высотой $\left(k_{\omega} a=0.1, k_{\omega} h=5\right)$. В этом случае диаграмма направленности сплющивается вдоль оси $O z$. При дальнейшем увеличении высоты также появляются побочные лепестки.

Рис. 3, с характеризует генерацию от торцевой поверхности цилиндрической частицы при большом значении радиуса основания $\left(k_{\omega} a=2, k_{\omega} h=0.1\right)$. На диаграмме также выделяются два главных лепестка, а при дальнейшем увеличении радиуса основания и множество побочных. На рис. 3, $d$ генерация уже происходит от торцевой поверхности цилиндрической частицы большой высоты $\left(k_{\omega} a=0.1, k_{\omega} h=5\right)$. При этом также наблюдается появление побочных лепестков диаграммы направленности.

Размеры частицы также влияют и на то, какая часть поверхности (торцы или боковая поверхность) цилиндра будет вносить решающий вклад в генерацию. На рис. $3, e$ изображена диаграмма направленности от всей поверхности цилиндрической частицы $\left(k_{\omega} a=0.1, k_{\omega} h=0.1\right)$. При этом ее форма больше соответствует генерации от торцевой поверхности (рис. 2,e), чем от боковой (рис. 2,a) следовательно, именно мощность генерации от торцевой поверхности при таких размерах преобладает над мощностью генерации от боковой поверхности. Несложно проверить, что увеличение радиуса основания ведет к возрастанию вклада торцевых поверхностей 


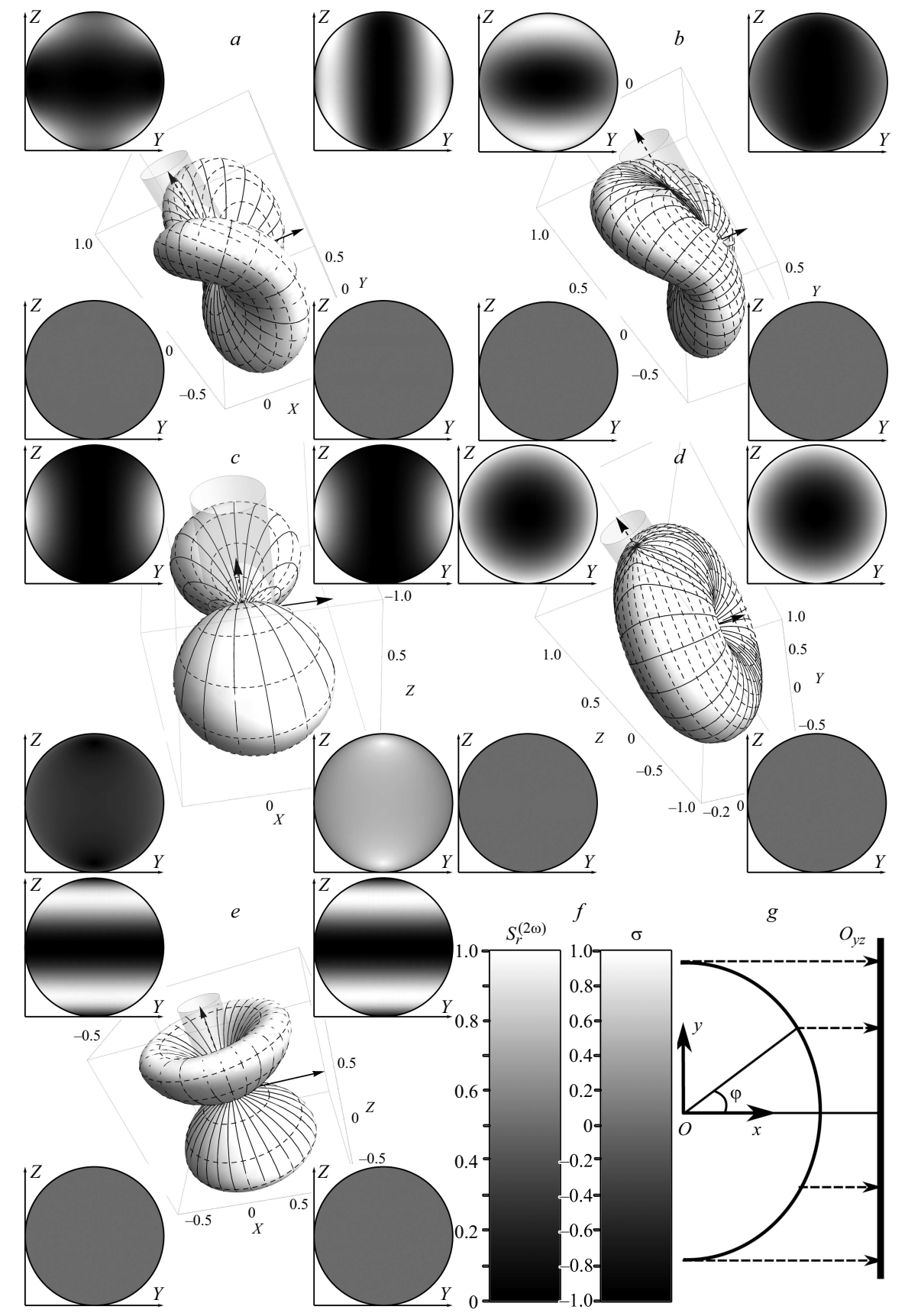

Рис. 2. Трехмерные нормированные диаграммы направленности для генерации второй гармоники от поверхности цилиндрической частицы при различных значениях коэффициентов анизотропии. Положение цилиндрической частицы схематично обозначено прозрачным цилиндром. Сплошная стрелка указывает на направление волнового вектора падающей волны. Пунктирная стрелка направлена вдоль большой полуоси эллипса поляризации падающей волны. Проекции больших и меньших полуосей эллипса поляризации генерируемых волн на поверхность диаграммы направленности являются касательными к сплошным и штриховым линиям на ее поверхности соответственно. Вверху на правом (левом) графике показаны проекции (как показано схематично на pис. $2, g)$ диаграммы на плоскость $O y z$ для значений азимутального угла $-\pi / 2<\varphi<\pi / 2(\pi / 2<\varphi<3 \pi / 2)$. Согласно легенде, на рис. $2, f$ белому цвету соответствует наибольшая мощность генерируемого излучения, а черному - наименьшая. Степень эллиптичности генерируемого излучения характеризуется графиками в левом и правом нижних углах. Правила проецирования на этих графиках те же. Белому и черному цвету соответствуют право и лево циркулярно поляризованные волны (соответствующая легенда представлена на рис. 2,f). Рис. 2, $a-d$ характеризуют генерацию от боковой поверхности, а рис. 2,e - от торцевых. Коэффициент, характеризующий дисперсию, $\xi=1$ для всех построенных диаграмм. Остальные параметры построения для каждой из диаграмм: $a-k_{\omega} a=0.1, k_{\omega} h=0.1, \sigma=0.5, \theta_{i n}=\pi / 2, \varphi_{\text {in }}=0, \chi_{1}^{(2)} \neq 0, \chi_{2-4}^{(2)}=0 ; b-k_{\omega} a=0.1, k_{\omega} h=0.1, \sigma=0.5$, $\theta_{i n}=\pi / 2, \varphi_{\text {in }}=0, \chi_{2}^{(2)} \neq 0, \chi_{1,3,4}^{(2)}=0 ; c-k_{\omega} a=0.1, k_{\omega} h=0.1, \sigma=0.5, \theta_{i n}=\pi / 2, \varphi_{i n}=0, \chi_{3}^{(2)} \neq 0, \chi_{1,2,4}^{(2)}=0 ; d-k_{\omega} a=0.1$, $k_{\omega} h=0.1, \sigma=0.5, \theta_{i n}=\pi / 2, \varphi_{i n}=0, \chi_{4}^{(2)} \neq 0, \chi_{1-3}^{(2)}=0 ; e-k_{\omega} a=0.1, k_{\omega} h=0.1, \sigma=0.5, \theta_{i n}=\pi / 2, \varphi_{i n}=0, \chi_{1,2}^{(2)} \neq 0, \chi_{3,4}^{(2)}=0$. 


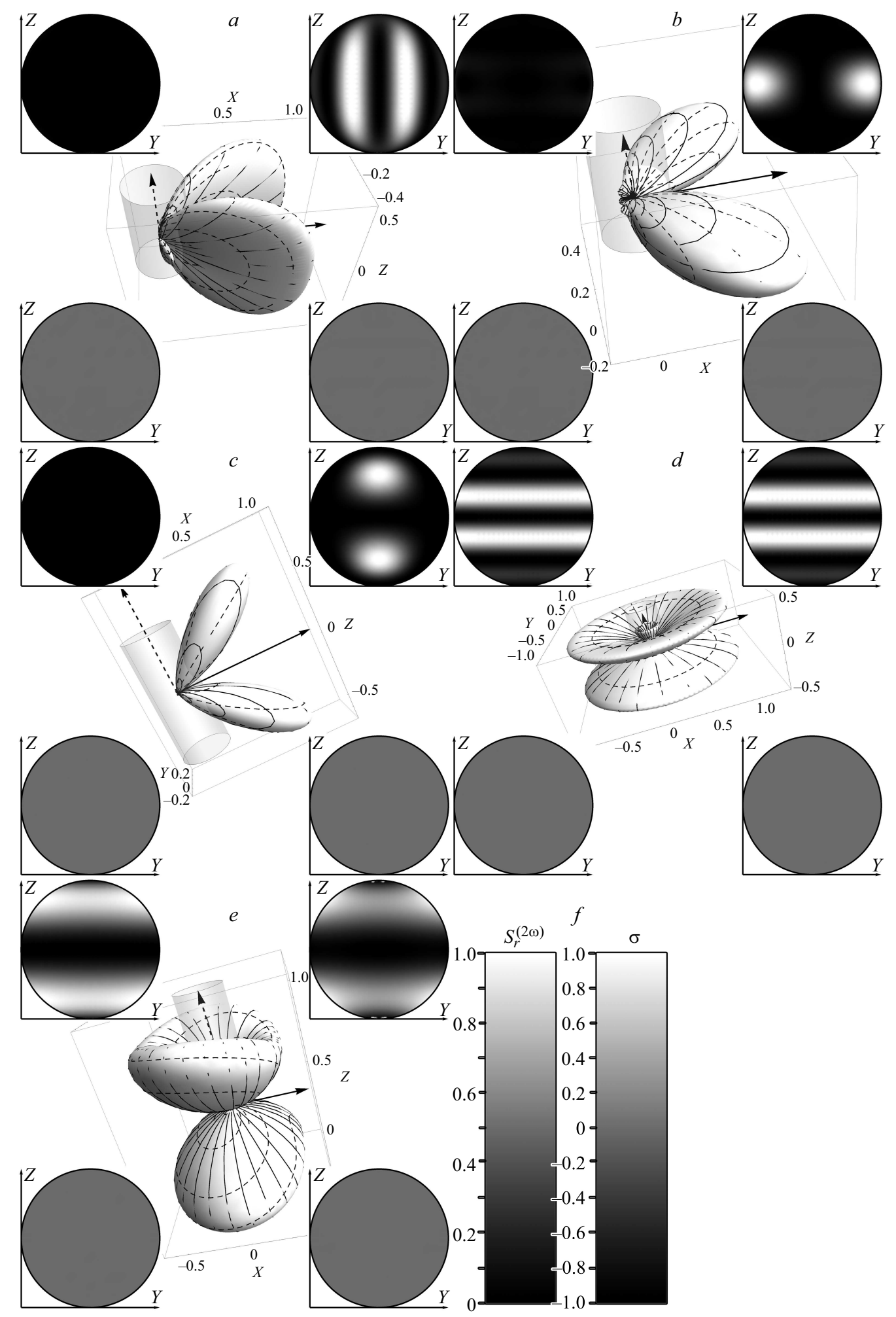

Рис. 3. Трехмерные нормированные диаграммы направленности для генерации второй гармоники от поверхности цилиндрической частицы при различных размерах частицы. Рис. $3, a, b$ характеризуют генерацию от боковой поверхности частицы, рис. $3, c, d-$ от торцевых, а рис. $3, e-$ от всей поверхности цилиндра. Обозначения аналогичны указанным в подписи рис. 2. Для всех диаграмм $\xi=1$; остальные параметры построения: $a-k_{\omega} a=2, k_{\omega} h=0.1, \sigma=0.5, \theta_{i n}=\pi / 2, \varphi_{i n}=0, \chi_{1}^{(2)} \neq 0, \chi_{2-4}^{(2)}=0 ; b-k_{\omega} a=0.1$, $k_{\omega} h=5, \sigma=0.5, \theta_{i n}=\pi / 2, \varphi_{i n}=0, \chi_{1}^{(2)} \neq 0, \chi_{2-4}^{(2)}=0 ; c-k_{\omega} a=2, k_{\omega} h=0.1, \sigma=0.5, \theta_{i n}=\pi / 2, \varphi_{i n}=0, \chi_{1}^{(2)} \neq 0, \chi_{2-4}^{(2)}=0$; $d-k_{\omega} a=0.1, k_{\omega} h=5, \sigma=0.5, \theta_{i n}=\pi / 2, \varphi_{i n}=0, \chi_{1}^{(2)} \neq 0, \chi_{2-4}^{(2)}=0 ; e-k_{\omega} a=0.1, k_{\omega} h=0.1, \sigma=0.5, \theta_{i n}=\pi / 2, \varphi_{i n}=0$, $\chi_{1}^{(2)} \neq 0, \chi_{2-4}^{(2)}=0$. Легенда представлена на рис. $3, f$. 


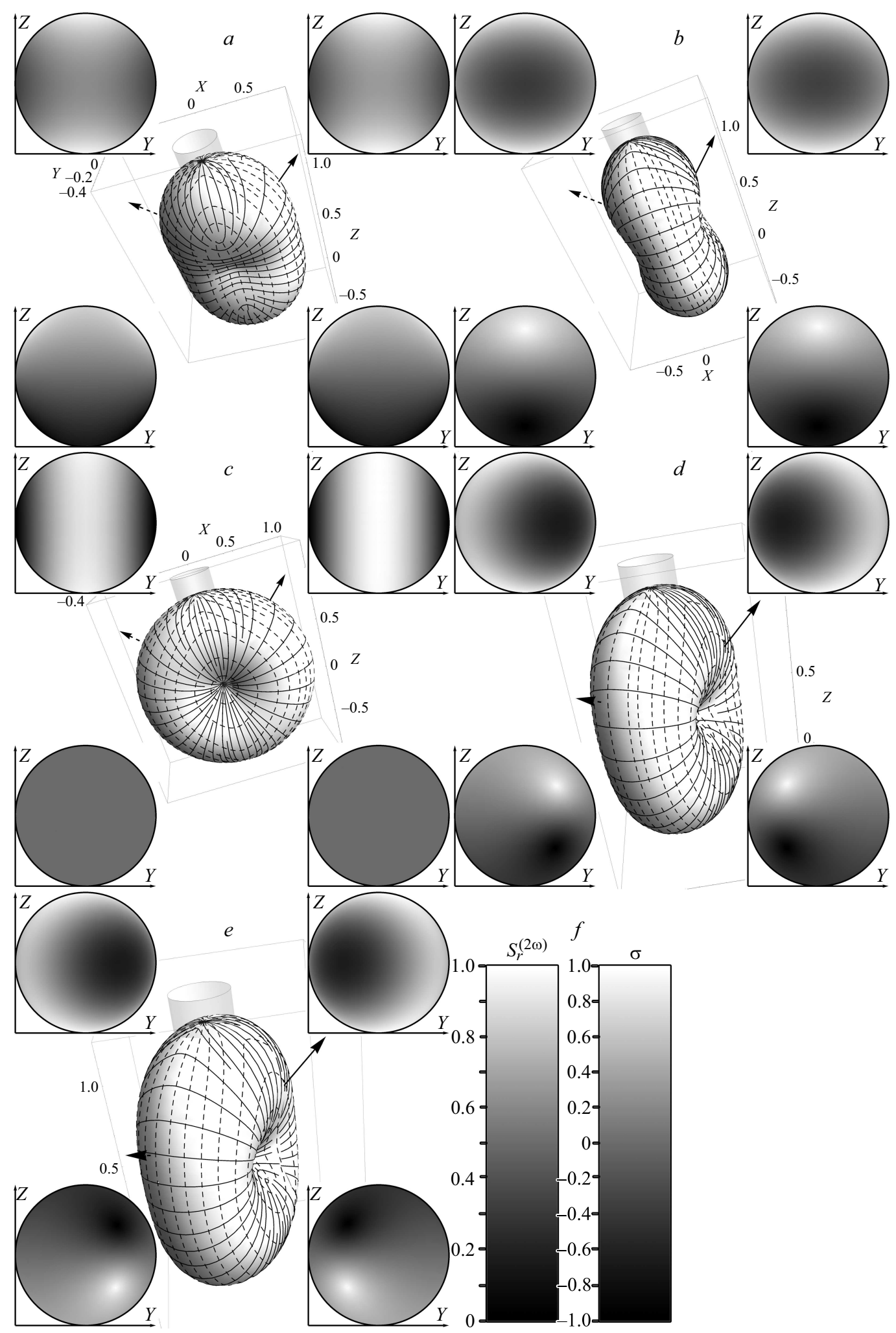

Рис. 4. Трехмерные нормированные диаграммы направленности для генерации второй гармоники от боковой поверхности цилиндрической частицы при различных поляризациях и углах падения. Обозначения аналогичны указанным в подписи рис. 2. Для всех диаграмм $\xi=1$. Остальные параметры построения: $a-k_{\omega} a=0.1, k_{\omega} h=0.1, \sigma=0.5, \theta_{i n}=\pi / 4, \varphi_{\text {in }}=0, \chi_{4}^{(2)} \neq 0$, $\chi_{1-3}^{(2)}=0 ; b-k_{\omega} a=0.1, k_{\omega} h=0.1, \sigma=1, \theta_{i n}=\pi / 4, \varphi_{i n}=0, \chi_{4}^{(2)} \neq 0, \chi_{1-3}^{(2)}=0 ; c-k_{\omega} a=0.1, k_{\omega} h=0.1, \sigma=0, \theta_{i n}=\pi / 4$, $\varphi_{\text {in }}=0, \chi_{4}^{(2)} \neq 0, \chi_{1-3}^{(2)}=0 ; d-k_{\omega} a=0.1, k_{\omega} h=0.1, \sigma=0.5, \theta_{i n}=\pi / 4, \varphi_{i n}=\pi / 4, \chi_{4}^{(2)} \neq 0, \chi_{1-3}^{(2)}=0 ; e-k_{\omega} a=0.1, k_{\omega} h=0.1$, $\sigma=-0.5, \theta_{i n}=\pi / 4, \varphi_{i n}=\pi / 4, \chi_{4}^{(2)} \neq 0, \chi_{1-3}^{(2)}=0$. Легенда представлена на рис. $4, f$. 
в генерацию, а увеличение высоты частицы ведет к возрастанию вклада боковой поверхности частицы.

На рис. 4 отражено влияние остальных параметров задачи на генерацию: угла падения, эллиптичности, ориентации эллипса поляризации. На этот раз сравнение диаграммы построено для значения коэффициентов анизотропии $\chi_{4}^{(2)} \neq 0, \chi_{1-3}^{(2)}=0$. Сравнивая рис. $2, d$ и рис. $4, a$, видим, что изменение угла падения деформирует диаграмму направленности. Аналогичное явление наблюдается при изменении степени эллиптичности падающего излучения (рис. $4, a, b$ и $c$ ). Поворот плоскости поляризации падающего излучения также оказывает большое влияние на форму диаграммы направленности, что можно наблюдать, если сравнить рис. 4, $c$ и $d$. Изменение поляризации падающей волны с правой на левую не влияет на форму диаграммы направленности, но меняет поляризацию генерируемых волн на противоположную (рис. 4, $d$ и $e$ ).

Также на диаграммах направленности можно видеть выполнение отдельных свойств решения. Например, на диаграмме направленности на рис. 2, а показана генерация линейно поляризованного излучения. Генерация исключительно линейно поляризованного излучения от боковой поверхности цилиндрической частицы должна наблюдаться, когда падающая волна направлена перпендикулярно оси цилиндрической частицы $\left(\theta_{i n}=\pi / 2\right)$, а коэффициенты анизотропии $\chi_{2,3,4}^{(2)}$ равны нулю. Аналогично для рис. 2, $b$ : генерация линейно поляризованного излучения наблюдается от всей поверхности цилндрической частицы, когда значения коэффициентов анизотропии $\chi_{1,3,4}^{(2)}$ равны нулю.

Линейной поляризации излучения на рис. $2, e, 3, c, d$ соответствует следующее свойство: если коэффициенты $\chi_{3,4}^{(2)}$ равны нулю, то от торцов цилиндрической частицы генерируется исключительно линейно поляризованное излучение. Аналогично рис. $3, a, b, e$ соответствует свойство: если $\chi_{3,4}^{(2)}=0, \theta_{i n}=\pi / 2, \varphi_{\text {in }}=m \pi / 2, m-$ целое, то от всей поверхности цилиндрической частицы генерируется исключительно линейно поляризованное излучение. Линейная поляризация излучения на рис. 4, $c$ объясняется следующим свойством: если коэффициенты $\chi_{1-3}^{(2)}$ равны нулю, то при падении линейно поляризованного излучения на цилиндрическую частицу от ее поверхности генерируется исключительно линейно поляризованное излучение.

Направления, в которых отсутствует излучение, также наблюдаются на графиках. Например, на рис. 2, a наблюдается свойство: если $\theta_{i n}=\pi / 2$, то в направлениях $\theta=\pi / 2, \varphi=0, \pi$ генерация излучения от всей повехности цилиндрической частицы отсутствует. На рис. 2, $c$ излучение отсутствует в плоскости $O x z$, что объясняется свойством: если $\chi_{1,2,4}^{(2)}=0, \theta_{i n}=\pi / 2$, то излучение от боковой поверхности частицы отсутствует в направлениях, для которых $\varphi=0, \pi$. На рис. 2,e генерация не наблюдается в плоскости $O x y$, что явля- ется проявлением следующего свойства: если $\chi_{4}^{(2)}=0$, $\theta_{i n}=\pi / 2$, то генерация не наблюдается в плоскости, где $\theta=\pi / 2$.

Также на диаграммах направленности можно наблюдать выполнение математических свойств для $\mathbf{f}^{(2 \omega)}(\theta, \varphi)$ и $S_{r}^{(2 \omega)}(\theta, \varphi)$. На рис. $2, a-e$ выполняются свойства (7) и (8), благодаря чему наблюдается две зеркальные плоскости симметрии диаграмм направленности (плоскости $O x z$ и $O x y)$. Аналогичные свойства наблюдаются и на рис. $3,4$.

\section{Заключение}

Анализ аналитического решения задачи о генерации второй гармоники от поверхности цилиндрической частицы привел к обнаружению ряда математических свойств функций, характеризующих пространственное распределение поля второй гармоники как для генерации от боковой поверхности, так и для генерации от всей поверхности цилиндра. Некоторые из этих свойств можно наблюдать на диаграммах направленности генерируемого излучения в виде плоскостей и осей симметрии. Часто такие свойства являются следствием симметрии схемы задачи.

Графический анализ показал, что форма диаграммы направленности и поляризация генерируемого излучения существенно зависят от значений коэффициентов анизотропии при малых размерах цилиндрической частицы. Увеличение радиуса основания практически всегда ведет к выделению двух главных и множества побочных лепестков диаграммы направленности, а увеличение высоты частицы приводит к сплющиванию диаграммы направленности и появлению небольших побочных лепестков.

\section{Финансирование работы}

Работа выполнена при финансовой поддержке БРФФИ (грант по проекту Ф18М-026).

\section{Список литературы}

[1] Yang N., Angerer W.E., Yodh A.G. // Phys. Rev. Lett. 2001. V. 87. N 10. P. 103902. doi 10.1103/PhysRevLett.87.103902

[2] Martorell J., Vilaseca R., Corbalan R. // Phys. Rev. A. 1997. V. 55. N 6. P. $4520-4525$. doi 10.1103/PhysRevA.55.4520

[3] Shan J., Dadap J.I., Stiopkin I., Reider G.A., Heinz T.F. // Phys. Rev. A. 2006. V. 73. N 2. P. 023819. doi 10.1103/PhysRevA.73.023819

[4] Jen S.-H., Dai H.-L., Gonella G. // J. Phys. Chem. C. 2010. V. 114. N 10. P. 4302. doi 10.1021/jp910144c

[5] Капшай В.Н., Шамына А.А. // Опт. и спектр. 2017. T. 123. № 3. C. 416-429. doi 10.7868/S003040341709015X; Kapshai V.N., Shamyna A.A. // Opt. Spectrosc. 2017. V. 123. N 3. P. 440-453. doi 10.1134/S0030400X17090144 
[6] Шамына А.А., Капшай В.Н. // Опт. и спектр. 2018. Т. 124. № 1. C. 105-121. doi 10.21883/OS.2018.01.45366.176-17; Shamyna A.A., Kapshai V.N. // Opt. Spectrosc. 2018. V. 124. N 1. P. 103-120. doi 10.1134/S0030400X18010198

[7] Капшай В.Н., Шамына А.А. // Опт. и спектр. 2018. Т. 124. № 6. C. 795-803. doi 10.21883/OS.2018.06.46083.55-18; Kapshai V.N., Shamyna A.A. // Opt. Spectrosc. 2018. V. 124. N 6. P. 826-833. doi 10.1134/S0030400X18060115

[8] Шамына А.А., Капшай В.Н. // Опт. и спектр. 2018. T. 125. № 1. C. 71-78. doi 10.21883/OS.2018.07.46269.56-17; Shamyna A.A., Kapshai V.N. // Opt. Spectrosc. 2018. V. 125. N 1. P. 74-81. doi 10.1134/S0030400X1807024X 